AperTO - Archivio Istituzionale Open Access dell'Università di Torino

\title{
On Measuring Inequity in Taxation Among Groups of Income Units
}

\section{This is the author's manuscript}

Original Citation:

Availability:

This version is available http://hdl.handle.net/2318/138927

since 2017-05-16T19:43:48Z

Published version:

DOI:10.1111/roiw.12070

Terms of use:

Open Access

Anyone can freely access the full text of works made available as "Open Access". Works made available under a Creative Commons license can be used according to the terms and conditions of said license. Use of all other works requires consent of the right holder (author or publisher) if not exempted from copyright protection by the applicable law. 


\section{(3) \\ UNIVERSITÀ DEGLI STUDI DI TORINO}

This is an author version of the contribution published on:

Questa è la versione dell'autore dell'opera:

Maria Giovanna Monti, Simone Pellegrino and Achille Vernizzi, "On Measuring Inequity in Taxation Among Groups of Income Units", The Review of Income and Wealth, 2015, Vol. 61(1), pp. 43-58. DOI: 10.1111/roiw.12070.

The definitive version is available at:

La versione definitiva è disponibile alla URL:

http://onlinelibrary.wiley.com/doi/10.1111/roiw.12070/abstract 


\title{
On Measuring Inequity in Taxation Among Groups of Income Units
}

\author{
July $20^{\text {th }}, 2013$
}

\author{
Maria Giovanna Monti \\ Department of Economics, Management and Quantitative Methods, Università degli \\ Studi di Milano
}

Simone Pellegrino

Department of Economics and Statistics, Università di Torino, Corso Unione Sovietica 218 bis, 10134, Torino, Italy.

Phone: +39 011670 6060; Fax: +39 011670 6062; e-mail: simone.pellegrino@unito.it

\section{Achille Vernizzi}

Department of Economics, Management and Quantitative Methods, Università degli Studi di Milano

\begin{abstract}
In this paper a method for analysing the fairness of an income tax system when portioning the population into heterogeneous socio-economic groups is proposed. The equitable tax system is defined by the three axioms given by Kakwani and Lambert (1998) and, as they suggest, inequity is evaluated by the negative influences on the redistributive effect of the tax associated with axiom violations. Measuring the extent of axiom violations among households belonging to different groups, we improve the Kakwani and Lambert analysis, which is able to detect only the existence of overall inequities. We propose a method that allows for evaluation of the contribution of each group to the overall inequity. Moreover, the adopted method enables disentangling the directions of violations. The obtained results allow us to judge how axiom violations discriminate among groups in their reciprocal relationships. An application to the 2010 Italian income tax reveals that inequities disproportionately penalize the household typologies. More precisely, unfairness affects households with children more severely than the other household groups.
\end{abstract}

JEL Codes: $C 81, H 23, H 24$

Keywords: Micro-simulation Models, Personal Income Tax, Progressive Principle, Redistributive Effect, Re-ranking 


\section{Introduction ${ }^{1}$}

As Lambert (2004, p. 39) notes, income tax is particularly subject to criteria of equity, being the unique tax instrument through which the government directly approaches its citizens. Horizontal and vertical equity are two of the basic commands of social justice that have been applied to income tax, raising a number of issues (Aronson, Johnson and Lambert, 1994; Aronson and Lambert, 1994; Aronson, Lambert and Trippeer, 1999; Wagstaff et al., 1999; van de Ven, Creedy and Lambert, 2001; Hyun and Lim, 2005; Urban and Lambert, 2008, among others).

However, although the existing literature is very wide, it is not easy to check to what extent a tax system is fair. One example of this difficulty may be, for instance, the academic debate on the significance of horizontal equity itself. It is known that Kaplow (1989) and Kaplow and Shavell (2002) express reservations about its relevance, while Auerbach and Hassett (2002) and Dardanoni and Lambert (2001), among others, see the horizontal (in)equity notion as continuing to hold a firm grip on the assessment of fair tax policies.

In the above-cited papers, however, the individuals have to be identical in every respect but, possibly, income. Things become much more complicated if we assume that the economic units may also differ with respect to further attributes: for instance, in the case of households we can have differences in size or needs. In this paper, we consider a non-homogeneous population. Dealing with a population partitioned by groups, we try to evaluate to what extent a tax system violates equity principles through 'preferential' treatment of certain groups of income units in comparison to some other groups. To pursue this aim, we start by the axiomatic definition of an equitable tax system provided by Kakwani and Lambert (1998) (hereafter KL). This choice allows us to avoid the question of measuring explicitly horizontal inequity. In their article, KL define equity in the income tax system by means of three axioms designed in such a way as to be independent. The three negative influences associated with axiom violations provide a mean to assess the extent of the inequity. The authors perform an overall analysis on a population of income units made homogeneous by applying a proper equivalence scale.

${ }^{1}$ We wish to thank Paolo Liberati and Dino Rizzi as well as two anonymous Referees of this journal for their helpful comments. Usual disclaimers apply. 
The extent of each type of inequity is measured by particular re-ranking indices, which are obtained starting from Lorenz curves.

In contrast, we suppose a population partitioned into groups and define the Gini and concentration coefficients by differences between attributes related to pairs of income units. The values of the two coefficients are obtained by particular indicator functions. These definitions of the two coefficients lead to splitting the extents of the three inequity types into their within- and across-group components, both the components being re-ranking measures. As a consequence, the three across-group components are defined considering the contributions of group pairs to the overall across-group violations. We show that the proposed method allows for evaluation of the contribution of each single group to the overall inequity, and, what is more important, it allows judgement of how axiom violations discriminate among groups in their reciprocal relationships.

More specifically, the groups can be ordered by their own level of penalization; the direction of group penalization deriving from axiom violations, either when pairs of groups are considered or when a single group is compared with all the others, can then be detected.

No particular further effort is required to decompose the redistributive effect of taxes when the same method presented for the re-ranking indices is applied. This permits the obtaining of some other results. We can assess more precisely whether axiom violations undermine unfairness largely either within groups or across groups. Moreover, by means of the decomposition of the redistributive effect, it is possible to verify how a tax system distributes its possible inefficiencies through different groups of income units.

The results are applied to the Italian Personal Income Tax. We recall that the income unit of the Italian Personal Income Tax is the individual. In terms of the present tax law, family charges are taken into account by using two sets of tax credits: a) tax credits for items of expenditure related to children, such as tuition fees; b) tax credits for working status and dependent relatives (children and spouse, as well as other individuals), which decrease with respect to the taxpayer's gross income and become zero above a specific threshold. 
We investigate the fairness of the Italian tax system with respect to households with different characteristics, using, as input data, those provided by the Bank of Italy (2012) in its Survey on Household Income and Wealth in the 2010 fiscal year.

The remainder of the paper is structured as follows. Section 2 presents the basic model proposed by KL. Section 3 presents the within- and across-group decompositions of the inequity associated with axiom violations. In the same section, the measure of the contribution of each group to the overall level of inequity is presented. In Section 4, we recall the microsimulation model employed for the empirical analysis and present the empirical strategy; then the analytical instruments, which are introduced in Section 3, are applied to the Italian Personal Income Tax. Section 5 concludes.

\section{The Basic Model}

KL (p. 370) state that a tax system should respect the following three axioms in order to be equitable: tax should increase monotonically with respect to people's ability to pay (Axiom 1); richer people should pay taxes at higher rates (Axiom 2); no re-ranking should occur in people's living standards (Axiom 3). The three axioms can be formally expressed as follows: let $X, Y, T$ and $A$ be the pre- and post-tax income distribution, the tax liability distribution and the average tax rate distribution, respectively. For each pair of income units $(\{i, j\}, i, j=1,2, \ldots, H)$ it must hold:

Axiom 1: $x_{i} \geq x_{j} \Rightarrow t_{i} \geq t_{j}$; this is a requirement of minimal progression. KL (p. 370) observe that "because the inequalities are weak, horizontal equity is part of this Axiom". Axiom 2: $x_{i} \geq x_{j}$ and $t_{i} \geq t_{j} \Rightarrow a_{i} \geq a_{j}$; this is the content of the progressive principle. Axiom 3: $x_{i} \geq x_{j}$ and $t_{i} \geq t_{j}$ and $t_{i} / x_{i} \geq t_{j} / x_{j} \Rightarrow y_{i} \geq y_{j}$; this is a vertical restriction ruling out too much progressivity.

A violation of Axiom 1 automatically entails a violation of Axiom 2, although not necessarily the other way round. Moreover, Axiom 3 can be violated only if Axiom 2 (and consequently Axiom 1) holds.

The violations of the three axioms are detected by determining whether the ordering of the distribution of $T, A$ and $Y$ are the same as that of $X$. Thus, the following three reranking indexes are suggested (KL, p. 373): 
i) $R_{T}=G_{T}-C_{T \mid X}$; ii) $R_{A}=\left(G_{A}-C_{A \mid X}\right)$; iii) $R_{Y}=G_{Y}-C_{Y \mid X}$.

where $G_{X}, G_{T}, G_{A}$ and $G_{Y}$ denote the Gini coefficient for pre-tax incomes, tax liabilities, average tax rates and post-tax incomes, respectively; $C_{T \mid X}, C_{A \mid X}$ and $C_{Y \mid X}$ denote the corresponding concentration coefficients when $T, A$ and $Y$ are ordered by pretax income. ${ }^{2}$ Consequently, the axiom violations are identified by $R_{T}>0$ for Axiom 1 , by $R_{A}>0$ for Axiom 2 and by $R_{Y}>0$ for Axiom 3. The departure from equity of the income tax system is evaluated starting from a particular decomposition of the redistributive effect $R E=G_{X}-G_{Y}$, which, according to Kakwani (1984, p. 165), may be written as

$$
R E=\tau P-R_{Y},
$$

where: $P=C_{T \mid X}-G_{X}$ is the Kakwani progressivity index and $\tau$ is the ratio between the total amount of the tax and the total amount of the post-tax income. Then equation (2) is decomposed as:

$$
R E=\tau\left[P+R_{A}\right]-S_{1}-S_{2}-S_{3} .
$$

In Equation (3), $\tau\left[P+R_{A}\right]$ may be thought as a measure of the implicit or potential equity, while

$$
S_{1}=\tau R_{T \mid X}, S_{2}=\tau\left(R_{A}-R_{T}\right) \text { and } S_{3}=R_{Y}
$$

provide an easy check on axioms 1-3. Their amounts indicate the extent of the departures from equity evaluated as losses of redistributive effect. ${ }^{3}$

\section{Within and Across-group Decompositions of the Redistributive Losses}

Consider a population of income units which can be gathered into $L$ groups, $H_{d}$ being the number of units in group $d(d=1,2, \ldots, L)$. A weight $p_{d, i}\left(d=1,2, \ldots, H_{d}\right)$ is associated with each income unit with $\sum_{i=1}^{H_{d}} p_{d, i}=N_{d}$ and $\sum_{d=1}^{L} N_{d}=N .{ }^{4}$ The weight system depends on the scale coefficient associated with each unit, and when dealing with a sample, it also depends on the sample representativeness of the unit. Let $Z$ be a

\footnotetext{
${ }^{2} \mathrm{KL}$ performe their analysis defining Gini and concentration coefficients by Lorenz curves. In particular, they defined the concentration curve $L_{Z \mid X}$ as the Lorenz curve for an attribute $\mathrm{Z}$ of income units, when these are ordered by the attribute $X$.

${ }^{3}$ In order to exactly apply the KL Axiom 2 statement, Pellegrino and Vernizzi (2012) propose a different approach for its measurement. However, in this paper the absolute extent of Axiom 2 violations does not strictly matter; as a consequence, we chose to employ the method originally suggested by KL.

${ }^{4}$ If we associate $p_{d, i}=1$ to each income unit, $N_{d}$ and $N$ denote the number of income units in group $d$ and in the overall population, respectively.
} 
generic attribute characterizing the income units, we denote by $z_{d, i}$ and $\mu_{Z}$ the level of the attribute of $Z$ associated with the unit $i$ belonging to the group $d$ and the overall average in the population. We compute the Gini coefficient by the Gini's Mean Difference approach. In so doing, the overall Gini coefficient can be defined as a function of the differences between attribute levels and of the indicator function $I_{i-j}^{Z}$ :

$$
G_{Z}=\frac{1}{2 \mu_{Z} N^{2}} \sum_{d=1}^{L} \sum_{m=1}^{L} \sum_{i=1}^{H_{d}} \sum_{j=1}^{H_{m}}\left(z_{d, i}-z_{m, j}\right) p_{d, i} p_{m, j} I_{i-j}^{Z}, I_{i-j}^{Z}=\left\{\begin{array}{cc}
1: & z_{d, i} \geq z_{m, j} \\
-1: & z_{d, i}<z_{m, j}
\end{array} .\right.
$$

Analogously, the concentration coefficient $C_{Z \mid X}$ for the same attribute $\mathrm{Z}$ is defined as in (6), when the income units are lined up by non-decreasing order of another attribute $X$ :

$$
C_{Z \mid X}=\frac{1}{2 \mu_{Z} N^{2}} \sum_{d=1}^{L} \sum_{m=1}^{L} \sum_{i=1}^{H_{d}} \sum_{j=1}^{H_{m}}\left(z_{d, i}-z_{m, j}\right) p_{d, i} p_{m, j} I_{i-j}^{Z \mid X}, I_{i-j}^{Z \mid X}=\left\{\begin{array}{c}
1: x_{d, i}>x_{m, j} \\
-1: x_{d, i}<x_{m, j} \\
I_{i-j}^{Z}: x_{d, i}=x_{m, j}
\end{array} .\right.
$$

From expressions (5) and (6), it derives that the re-ranking index for $Z$ with respect to $X$, $R_{Z}=G_{Z}-C_{Z \mid X}$, is given by:

$$
R_{Z}=\sum_{d=1}^{L} \sum_{m=1}^{L} R_{Z}^{d, m}
$$

with

$$
R_{Z}^{d, m}=\frac{1}{2 \mu_{Z} N^{2}} \sum_{i=1}^{H_{d}} \sum_{j=1}^{H_{m}}\left(z_{d, i}-z_{m, j}\right) p_{d, i} p_{m, j}\left(I_{i-j}^{Z}-I_{i-j}^{Z \mid X}\right) .
$$

From expressions (8), it follows that $R_{Z}^{d, d}$ defines the contribution of group $d$ to the overall within group re-ranking. This contribution is a function of the re-ranking within the group $d$ and of strictly positive weighting functions representing the share of income units and the share of post-tax income attributed to group $d$. The contribution of the group pair $d, m$ to the overall across group re-ranking, is represented by $\left(R_{Z}^{d, m}+R_{Z}^{m, d}\right)$ and it will be denoted by $R_{Z}^{d \cup m}=R_{Z}^{m \cup d} . R_{Z}^{d \cup m}$ is a function of the re-ranking between individuals of group $d$, compared with individuals of group $m$ (or vice-versa) and of the two functions, which represent the shares related to the two groups. 
It can be shown that the re-ranking $R_{Z}$ decomposes into two re-ranking measures: ${ }^{5}$ the measure of re-ranking within each group, $R_{Z}^{W}$, and the measure of re-ranking across groups, $R_{Z}^{A G}$, which evaluates the re-ranking among units belonging to different groups. Then, from the above given definitions, we write ${ }^{6}$

$$
R_{Z}^{W}=\sum_{d=1}^{L} R_{Z}^{d, d} \text { and } R_{Z}^{A G}=\sum_{d=1}^{L} \sum_{m=d+1}^{L} R_{Z}^{d \cup m}
$$

Decompositions (9) can be applied to decompose the losses of redistributive effects defined in (4) into their within- and across-group components. Denoting by $S_{1}^{W}, S_{2}^{W}$ and $S_{3}^{W}$, and by $S_{1}^{A G}, S_{2}^{A G}$ and $S_{3}^{A G}$ the within- and the across-group components of $S_{1}, S_{2}$, and $S_{3}$, one writes:

$$
\begin{aligned}
& S_{1}=S_{1}^{W}+S_{1}^{A G}, S_{1}^{W}=\tau R_{T}^{W} \text { and } S_{1}^{A G}=\tau R_{T}^{A G} ; \\
& S_{2}=S_{2}^{W}+S_{2}^{A G}, S_{2}^{W}=\tau\left(R_{A}^{W}-R_{T}^{W}\right) \text { and } S_{2}^{A G}=\tau\left(R_{A}^{A G}-R_{T}^{A G}\right) ; \\
& S_{3}=S_{3}^{W}+S_{3}^{A G} \text { where } S_{3}^{W}=R_{Y}^{W} \text { and } S_{3}^{A G}=R_{Y}^{A G} .
\end{aligned}
$$

The contributions of group $d(d=1,2, \ldots, L)$ to the overall within effect are given by

$$
S_{1}^{d}=\tau R_{T}^{d, d}, S_{2}^{d}=\tau\left(R_{A}^{d, d}-R_{T}^{d, d}\right), S_{3}^{d}=R_{Y}^{d, d}
$$

and the ratios $\left(S_{s}^{d} / S_{s}\right) \%(s=1,2,3)$ are their normalized measures.

Considering the overall across group effect, expression (9) allows to define the joint contributions of groups $d$ and $m(d, m=1,2, \ldots, L ; d \neq m)$ as in (12)

$$
S_{1}^{d \cup m}=\tau R_{T}^{d \cup m}, S_{2}^{d \cup m}=\tau\left(R_{A}^{d \cup m}-R_{T}^{d \cup m}\right), S_{3}^{d \cup m}=R_{Y}^{d \cup m},
$$

It is clear from (12) that the problem of evaluating the contribution of a single group to overall across group effect arises. We will show that the ratio $\left(S_{s}^{d \cup} / 2 S_{s}\right) \%$ is a normalized measure of the contribution of the single group $d$ to the loss of redistributive effect due to the axiom $s(s=1,2,3)$ violation. The term $S_{s}^{d \cup \bullet}$ represents the overall axiom $s$ violations, which involve group $d$ with all the other $L-1$ groups.

Let us considered the structure of re-ranking between two groups.

\footnotetext{
${ }^{5}$ See Monti, Mussini and Vernizzi (2010) on the issue of re-ranking decomposition.

${ }^{6}$ Expressions in (9) are strictly linked with the Dagum (1997) decomposition of the Gini coefficient as discussed in Monti (2008). It is worth noting that an analogous decomposition has been recently formalized by Ebert (2010).
} 
Re-rankings across two groups, $d$ and $m$, can be observed in two different situations: $i$ ) $x_{d, i}<x_{m, j}$ and $z_{d, i}>z_{m, j}$;ii) $x_{d, i}>x_{m, j}$ and $z_{d, i}<z_{m, j}$. In $\left.i\right)$ the rank of the income unit $i$ belonging to group $d$ is lower than that of $j$ belonging to $m$ in $X$ distribution, and it is greater in $Z$ distribution; in $i i)$ the situation is reversed: the rank of the income unit $i$, belonging to group $d$, is greater than that of $j$, belonging to $m$ in $X$ distribution and it is lower in $Z$ distribution. Obviously, the re-ranking impact on the two income units is different in the two cases. In $i$ ), the income unit of $d$ goes beyond the unit of $m$ in the ranking of $Z$, when this ranking is compared with the ranking of $X$; we denote changes like this by $d \rightarrow m$, the direction of the arrow indicating the direction of the overtaking. In ii) the income unit of $m$ overtakes income unit of $d$; we denote these changes by $m \rightarrow d$. The (unfair) shifts of $d \rightarrow m$ favour $d$ if $Z$ is a desirable attribute (income), whilst they are against $d$ and in favour of $m$ if $Z$ is an unpleasant attribute (tax or tax rate). The last remark has to be reversed in case ii). Formally, in equation (8) one has: ${ }^{7}$

- $\quad$ case $i):\left(I_{i-j}^{Z}-I_{i-j}^{Z \mid X}\right)=2$, we denote by $R_{Z}^{d \rightarrow m}$ the weighted sum of the differences that in (8) are positive and then associated with 2.

- case $i i):\left(I_{i-j}^{Z}-I_{i-j}^{Z \mid X}\right)=-2$, we denote by $R_{Z}^{m \rightarrow d}$ the weighed sum of the differences that in (8) are negative and then associated with -2 .

The contribution $R_{Z}^{d \cup m}$ of the two groups $d$ and $m$ to the across-group re-ranking $R_{Z}^{A G}$ is then decomposed as:

$R_{Z}^{d \cup m}=R_{Z}^{d \rightarrow m}+R_{Z}^{m \rightarrow d}$.

The overall across group re-ranking, $R_{Z}^{A G}$, rewrites as

$$
R_{Z}^{A G}=\sum_{d=1}^{L} R_{Z}^{d \rightarrow \bullet}=\sum_{d=1}^{L} R_{Z}^{\bullet \rightarrow d}
$$

where $R_{Z}^{d \rightarrow \bullet}=\sum_{m=1, d \neq m}^{L} R_{Z}^{d \rightarrow m}$ represents the contribution to overall re-ranking depending on overtaking of income units of group $d$ on income units belonging to each other group, and $R_{Z}^{\bullet \rightarrow d}=\sum_{m=1, d \neq m}^{L} R_{Z}^{m \rightarrow d}$ represents the contribution to overall reranking given by overtaking of all other groups on group $d$.

\footnotetext{
${ }^{7}$ If both in the $Z$ and $X$ distribution the rank of $i$ is greater (lower) than the rank of $j$, no re-ranking occurs and $\left(I_{i-j}^{Z}-I_{i-j}^{Z \mid X}\right)=0$.
} 
Applying these results, we are able to write the contributions to the losses of the redistributive effect due to overtaking of group $d$ on group $m$ as:

$S_{1}^{d \rightarrow m}=\tau R_{T}^{d \rightarrow m} ; S_{2}^{d \rightarrow m}=\tau\left(R_{A}^{d \rightarrow m}-R_{T}^{d \rightarrow m}\right) ; S_{3}^{d \rightarrow m}=R_{Y}^{d \rightarrow m}$.

The contributions to the losses of the redistributive effect due to overtaking of group $d$ on all the other groups, and the contribution due to overtaking of each other group on group $d$, are given by

$$
S_{s}^{d \rightarrow \bullet}=\sum_{m=1, m \neq d}^{L} S_{s}^{d \rightarrow m}, S_{s}^{\bullet \rightarrow d}=\sum_{m=1, m \neq d}^{L} S_{s}^{m \rightarrow d}, \quad s=1,2,3 .
$$

The overall loss of redistributive effect that involve the single group $d$ when this group is compared with all the other $L-1$ groups is then

$$
S_{s}^{d \cup \bullet}=S_{s}^{d \rightarrow \bullet}+S_{s}^{\bullet \rightarrow d}, \quad s=1,2,3 .
$$

It is easy to see that $\sum_{d=1}^{L} S_{s}^{\bullet \rightarrow d}=\sum_{d=1}^{L} S_{s}^{d \rightarrow \bullet}$, and that both the sums are equal to $S_{s}^{A G}$, so that $\sum_{d=1}^{L} S_{s}^{d \cup \bullet}=2 S_{s}^{A G}$. This leads to suggest the ratio $\left(S_{s}^{d \cup \bullet} / 2 S_{s}\right) \%$ as a measure of the contribution of group $d$ to the loss of redistributive effect due to axiom $s$ violation.

Analogous decompositions apply to the redistributive effect of taxes. Two measures can be defined: the measure of the contribution to the overall actual redistributive effect due to tax effects within group $d, R E^{d}$, and the contribution due to tax effects between groups $d$ and $m, R E^{d \cup m} .^{8}$ The ratios between losses of redistributive effect and the correspondent redistributive effects allows detection of whether axiom violations undermine unfairness to a greater extent, either within groups or across groups, and to verify how a tax system distributes its possible inefficiencies through different groups of income units.

We conclude this section by observing that the Gini's Mean Difference approach permits a definition of the Gini coefficient and of all its components as a weighted sum of differences. In our case, this possibility builds a bridge between the measures we propose and some measures suggested by Duclos (2000) in order to evaluate the extent of some individual perceptions as relative deprivation, ill-fortune and resentment. ${ }^{9}$ For

\footnotetext{
${ }^{8}$ Details on $R E$ decompositions are available on request.
}

${ }^{9} \mathrm{We}$ thank an anonymous referee for this suggestion. 
instance, Duclos (2000, p.142) measures individual fiscal ill fortune as "the sum of the incomes of those individuals by whom they are overtaken minus the sum of the incomes of those they succeed in outranking". It is evident that, given this definition, and writing re-ranking as in (7) with $Z=Y$, no further efforts are required to define re-ranking as a weighted average of fiscal ill fortune in the population. The links between the results presented here and some measures derived from the deprivation literature (more or less directly) could be object of further researches.

\section{Data, Empirical Strategy and Results}

\subsection{Data and Empirical Strategy}

In order to apply our results we use as input data those provided by the Bank of Italy in its Survey on Household Income and Wealth (hereafter SHIW) published in 2012. This contains information on household post-tax income and wealth in the year 2010, covering 7,951 households, and 19,836 individuals. The sample is representative of the Italian population, which is composed of about 24 million households and 60 million individuals. As the SHIW provides only information on each individual's PIT net income, we estimate the PIT gross income for each taxpayer. The model we employ is an updated version of the model described in Pellegrino et al. (2011). Considering the income units, results concerning the gross income as well as tax liability distribution are very close to the Ministry of Economics and Finance's official statistics (2011).

Given that our theoretical results focus on a non-homogeneous population, we split Italian households into seven groups, according to their composition: 1) singles $(24.9 \%$ of total households);2) couples without children $(23.3 \%)$; 3) couples with one child $(16.1 \%) ; 4)$ couples with two children $(16.9 \%) ; 5)$ couples with three or more children $(4.9 \%)$; 6) parent alone with one or more children $(6.9 \%) ; 7)$ all other household typologies (7.1\%). Groups 3, 4, 5 and 6 are characterized by the presence of parents with children, groups 1 and 2 are characterized by the presence of sole adults and group 7 is a residual group. The choice of these groups is coherent with both the kind of decomposition we are going to apply, and the structure of Italian personal income tax, which provides income-related tax credits for earned income and dependent individuals within the household. This partition allows for an evaluation of the extent to which the 
Italian tax system considers the decreasing of the tax capability that occurs when the same income has to be shared by a different number of persons.

To perform the analysis, nominal incomes have to be transformed into equivalent incomes, and thus a proper equivalence scale has to be applied. The use of an equivalence scale is always necessary for across-group comparisons. In our case the scale is necessary also in the within-group analysis, because within each household typology the characteristics of the group units can be dissimilar. For instance, considering the typologies of family with children, children's ages can be different and the number of income earners can be unequal. Even dealing with singles, the working status of the group units can be different.

We choose to apply the equivalence scale $E S$ adopted by KL (p. 376) and defined as

$$
E S=\left(a+0.2 c_{1}+0.4 c_{2}+0.7 c_{3}\right)^{0.8}+0.1 w
$$

where $a$ is the number of adults within the households, $c_{1}$ is the number of children aged 5 years or less, $c_{2}$ is the number of children aged between 6 and 14 years, $c_{3}$ is the number of children aged between 15 and 17 years, $w$ is the number of employees or self-employed within the households, and 0.8 is the parameter that indicates the economies of scale attached to the equivalence scale.

This scale presents some advantages: it takes into account the age differences of the children, the number of parents, and the number of income earners. $E S$ introduces also economies of scale. ${ }^{10}$ Nevertheless, we remark that whatever the scale may be, its application can only partially mitigate within-group heterogeneity and make acrossgroup incomes equivalent for comparison.

As observed by Ebert and Moyes (2000, p. 131-132), there are different strategies for the application of the equivalence scale. In the empirical analysis discussed in Section 4.2, we weigh the equivalent household incomes by the equivalence scale. However, our results are also confirmed when equivalent incomes are not weighed by the equivalence scale.

\footnotetext{
${ }^{10}$ Results here presented have been compared with those obtained applying the OECD scale. The results obtained with the OCSE
} scale are coherent with those here reported for KL's scale, even if with fewer remarked disproportions in violations. 


\subsection{Results}

The starting point of our analysis is the original KL overall decomposition of the redistributive effect. Results are presented in Table 1. Focusing on equivalent households, ${ }^{11}$ the Gini coefficient $(\times 100)$ for the pre-tax equivalent household distribution is 38.88 , whilst the Gini coefficient for the post-tax distribution is 33.70 . The overall redistributive effect, $R E$, is then 5.18. The potential redistributive effect that could be obtained if all inequities could be abolished is equal to 5.98. Therefore, total inequities reduce the potential redistributive effect by 0.80 . From Table 1, we can conclude that the total axiom violations are $15.42 \%$ of the actual redistributive effect.

\section{TABLE 1 ABOUT HERE}

The lowest loss is due to Axiom 3 violations: the re-ranking from pre- to post-tax income distribution causes a loss of potential redistributive effect which is just $1.55 \%$ of $R E$; the loss due to Axiom 2 violations is the greatest, being more than $10 \%$ of $R E$, whilst the weight of Axiom 1 violations is $3.6 \%$ of $R E .^{12}$ From these results, we can say that the Italian tax system does not cause an excessive undue re-ranking in transition from pre- to post-tax income distribution. Axiom 2 violations are the greatest source of loss in the potential redistributive effect; nevertheless, some caution is needed in evaluating its amount, as pointed out in footnote 4 .

Then, considering each Axiom violation separately, our main goal is to describe the distribution of axiom violations either among or within household typologies as well as to assess the impact of axiom violations on each group.

To pursue these objectives, we evaluate the contribution of each household typology to the overall axiom violations (Table 2). Then, using expressions (13)-(17), we improve our analysis by describing and interpreting the direction of axiom violations among household typologies (Tables 3, 4 and 5). Lastly, the intensity of violations is evaluated with respect to the actual redistribution performance (Table 6).

Let us begin by analyzing the content of Table 2 . The last three rows of this table allow for some preliminary comments. First, we observe that the within-group effect is

\footnotetext{
${ }^{11}$ The average tax rate is $20.12 \%$. The concentration coefficient for the net income distribution is 33.62 , whilst that on the tax debt
} distribution is 59.75. Then the Kakwani index is 20.88, whilst the Reynolds-Smolensky index is 5.26. 
smaller than the across-group effect; the share of the former is about $16 \%$ of the overall effect for each axiom, whilst the share of the latter is a bit less than $84 \%$. This is not a surprising result; as Frosini (2012, pp. 182-183) observes, the greater the group number is, the lesser the relative contribution is of the 'within' component. Moreover, the same three rows of the table show that, although the measures of the overall axiom violations are different (see Table 1), the contributions of the within- and across-group effects to the overall violation amount to almost the same share whatever axiom we are considering.

\section{TABLE 2 ABOUT HERE}

Ordering the contributions of each group in decreasing order, one observes that the same ordering holds when considering both the contributions to the within-typology violations $\left(S_{s}^{d} / S_{s}\right) \%$ and the contributions to the across-group violations $\left(S_{s}^{d \cup \bullet} / 2 S_{s}\right) \%$. We can see that the group 'couple with two children' presents the highest values, followed by the group 'couples without children'. Typologies 5, 6 and 7 give the lowest contributions. However, interpreting the figures presented in Table 2 requires some caution. We cannot forget that these figures depend both on the average income and on the number of households related to each typology. When weighted by the equivalence scale, the number of households belonging to the typology 'couples with two children' is the largest one, while that belonging to the typology 'parent alone with children' is the smallest. ${ }^{13}$ Moreover, as underlined in Section 4.1, Groups 5, 6 and 7 are characterized by the highest degree of heterogeneity. We are conscious of these limits. However, on the one hand, to exclude typologies has a reductive effect on the analysis, and on the other hand, it is quite impossible to disentangle share amounts from re-ranking amounts, especially when evaluating the contribution of a group to the across-group axiom violations.

To improve our investigation, we detect the direction of axiom violations by explicitly applying the results obtained in (15). Tables 3, 4 and 5 show the direction of the impact

\footnotetext{
${ }^{12}$ We observe that the same ranking of axiom violations is estimated by KL for the 1984 Australian income tax.
} 
of axiom violations when pairs of household typologies are considered and when a particular typology is compared with all the others. Shares do not play any role in the extent of the results presented in these tables.

Table 3 takes into account violations concerning the lack of the requirement of minimal progression. Considering the contribution to Axiom 1 violations, we can see, for instance, that only $18.96 \%$ of tax liability re-rankings penalizes 'singles', whilst $74.89 \%$ penalizes couples 'with two children'. The figures in the last column of Table 3 show that 'singles' and 'couples without children' are the least penalized typologies whilst 'couples with two' and 'three or more children' are the most penalized. These results do not vary considering Axiom 2 and Axiom 3 violations (Table 4 and 5) violations: the typologies 'couples with two' and 'three or more children' are always the most penalized by re-rankings, with respect to all the remaining typologies. The behaviour of the impact of the axiom violations on the groups does not change if we consider the relations across households belonging to two different typologies: the 'singles' appear to be the least penalized and 'couples with two' and 'three or more children' are still the most penalized.

\section{TABLE 3 ABOUT HERE}

\section{TABLE 4 ABOUT HERE}

\section{TABLE 5 ABOUT HERE}

This penalization registered for households with children depends on the present structure of the Italian PIT. Our analysis seems to point out that the system of tax credits for dependent relatives (children in particular) is not so generous as it should be in order to limit this unpleasant outcome. From this point of view, the increase of the tax credits values for children introduced by the 2013 tax law should be viewed as a tool that may reduce the discriminations pointed out in this analysis.

It is interesting to note that the percentage of contribution to the overall axiom violations for 'couples with three children', $\left(S_{s}^{5 \cup} \bullet / 2 S_{s}\right) \%$, is not too high (see Table 2).

\footnotetext{
${ }^{13}$ If we rank household groups according to their weighed frequencies, we have: Group 4 (11 million), Group 2 (10 million), Group 3 (9 million), Group 1 (6.2 million), Group 7 (4.4 million), Group 5 (3.7 million), and Group 6 (3.2 million).
} 
This should suggest that corrections of unfairness involving households with three children might be performed with marginal efforts.

We conclude our analysis by using the ratio between axiom violations and the correspondent redistributive effects to pursue two goals. Firstly, we would detect whether axiom violations undermine unfairness largely, either within groups or across groups. Then we would verify how a tax system distributes its possible inefficiencies through different groups of households. From Table 6, we can immediately observe that for each axiom, the differences between the across-group ratio $\left(S_{s}^{A G} / R E^{A G}\right)$ and the within-group ratio $\left(S_{s}^{W} / R E^{W}\right)$ are much lower than those existing between $\left(S_{s}^{A G} / S_{s}\right)$ and $\left(S_{s}^{W} / S_{s}\right)$. However, the across-group ratio for each axiom $s(s=1,2,3)$ remains greater than the within-group ratio: all the three ratios $\left(S_{s}^{A G} / R E^{A G}\right)$ are roughly 1.15 times greater than the corresponding $\left(S_{s}^{W} / R E^{W}\right)$. Consequently, when compared with the redistribution actually performed, overall across-group violations can be considered more severe than within-group ones.

We observe that, for each axiom $s=1,2,3$, the ratios $\left(S_{s}^{d \cup m} / R E^{d \cup m}\right) \%$ (Table 6) present the same ordering as the ratios $\left(S_{s} / R E\right) \%$ (Table 1). A deeper insight into Table 6 allows to note that the group of 'singles' is the household typology which presents the lowest within values $\left(S_{s}^{1} / R E^{1}\right) \%$ for each $s$, whilst 'parent alone with children' $(d=6)$ and 'other typologies $(d=7)$ show the greatest violations. Interpreting these results requires the same remarks as were introduced when analyzing the contribution of each household typology to the overall axiom violations (Table 2). They may depend partially on the high homogeneity of the 'singles' and on the relative lack of homogeneity characterizing the last two typologies. Among the typologies with children and both parents, the 'couples with three children' presents the highest ratio between axiom violations and redistributive effect.

The ratio orderings do not change if we look at the overall relations of one typology with all the others; the highest ratios $\left(S_{s}^{d \cup \bullet} / R E^{d \cup \bullet}\right) \%$ are again registered by the group 
'parent alone with children' and 'other typologies', whilst the lowest values are observed when considering 'singles' and 'couples without children'.

\section{TABLE 6 ABOUT HERE}

\section{Conclusion}

We have proposed an approach for analyzing the fairness of an income-tax system when population is partitioned into heterogeneous socio-economic groups. The extent of inequity is evaluated starting with the KL axiomatic definition of an equitable tax system. This gives three axioms for an income tax to be equitable. Axiom violations exert distinct negative influences on the redistributive effect of the tax and the authors measure these negative influences by three re-ranking indexes: the re-ranking index of taxes, tax rates and net incomes with respect to pre-tax incomes. KL's analysis is limited to overall indicators.

Our analysis improves the KL results by considering a non-homogeneous population and measuring the extent of axiom violations among units belonging to different groups. An overall analysis (according to the original KL approach) simply detects the existence of inequities. Our analysis evaluates the within- and across-group inequity and detects the direction of the group penalization deriving from axiom violations either when pairs of groups are considered or when a single group is compared with all the others. This allows us to calculate the contribution of each group to the overall inequity and to judge how axiom violations discriminate among groups in their reciprocal relations.

Using the data provided by the Bank of Italy (2012) in its Survey on Household Income and Wealth in 2010, we apply our theoretical method to the personal income taxation of this country. We split households into groups characterized by different compositions and we evaluate the impact of departures from equity due to axiom violations within groups, across groups and in each group. In particular, for what concerns across-group violations, we evaluate the extent of the direction of violations.

Even if overall across-group violations are only a little more severe than within-group ones, when related to the corresponding redistributive effect, using our method we 
highlight a disproportion in the direction of across-group violations. By evaluating redistributive losses for each group, and for every pair of groups, our results show quite clearly that inequity is not proportionally distributed among the different groups of households. This lack of proportionality penalizes households with children, which appear to be particularly disadvantaged with respect to other household typologies. 
Table 1: Overall RE decomposition for households (x 100)

\begin{tabular}{lccccccccc}
\hline \hline & $\begin{array}{c}\text { Pre-tax } \\
\text { income }\end{array}$ & $\begin{array}{c}\text { Post-tax } \\
\text { income }\end{array}$ & $\boldsymbol{R E}$ & $\begin{array}{c}\text { Potential } \\
\text { equity }\end{array}$ & $\begin{array}{c}\text { Axiom } \\
\mathbf{1}\end{array}$ & $\begin{array}{c}\text { Axiom } \\
\mathbf{2}\end{array}$ & $\begin{array}{c}\text { Axiom } \\
\mathbf{3}\end{array}$ & $\begin{array}{c}\text { Total } \\
\text { Axioms }\end{array}$ \\
\hline \hline KL's Decomposition & 38.88 & 33.70 & 5.18 & 5.98 & 0.19 & 0.53 & 0.08 & 0.80 \\
\hline & - & - & 100.00 & 115.42 & 3.60 & 10.27 & 1.55 & 15.42 \\
\hline \hline
\end{tabular}

Source: Our elaborations on SHIW 2012. 
Table 2: Decomposition of the loss of redistributive effect due to axiom violations (\% of correspondent overall $S_{\mathrm{s}}, s=1,2,3$ )

\begin{tabular}{rrrrrrrr}
\hline \hline$\left(S_{1}^{d} / S_{1}\right) \%$ & 1.10 & 4.76 & 3.67 & 5.07 & 0.45 & 0.39 & 0.67 \\
$\left(S_{2}^{d} / S_{2}\right) \%$ & 1.14 & 4.08 & 3.62 & 5.50 & 0.54 & 0.52 & 0.73 \\
$\left(S_{3}^{d} / S_{3}\right) \%$ & 0.95 & 4.76 & 3.93 & 5.10 & 0.41 & 0.38 & 0.64 \\
\hline \hline$\left(S_{1}^{d \bullet \bullet} / 2 S_{1}\right) \%$ & 11.29 & 18.06 & 15.77 & 18.90 & 6.28 & 5.88 & 7.72 \\
$\left(S_{2}^{d \cup \bullet} / 2 S_{2}\right) \%$ & 11.24 & 16.70 & 15.50 & 19.23 & 6.77 & 6.52 & 7.78 \\
$\left(S_{3}^{d \cup \bullet} / 2 S_{3}\right) \%$ & 10.99 & 17.92 & 16.11 & 19.15 & 6.48 & 5.67 & 7.54 \\
\hline \hline & & & & $\left(S_{1}^{A G} / S_{1}\right) \%=83.88$ & \\
$\left(S_{1}^{W} / S_{1}\right) \%=16.12$ & & & $\left(S_{2}^{A G} / S_{2}\right) \%=83.88$ & \\
$\left(S_{2}^{W} / S_{2}\right) \%=16.12$ & & & $\left(S_{3}^{A G} / S_{3}\right) \%=83.84$ & \\
$\left(S_{3}^{W} / S_{3}\right) \%=16.16$ &
\end{tabular}

Source: Our elaborations on SHIW 2012. 
Table 3: Axiom 1 - Comparisons of losses between group pairs and between one group and all the others (\% of the two contrasting directions)

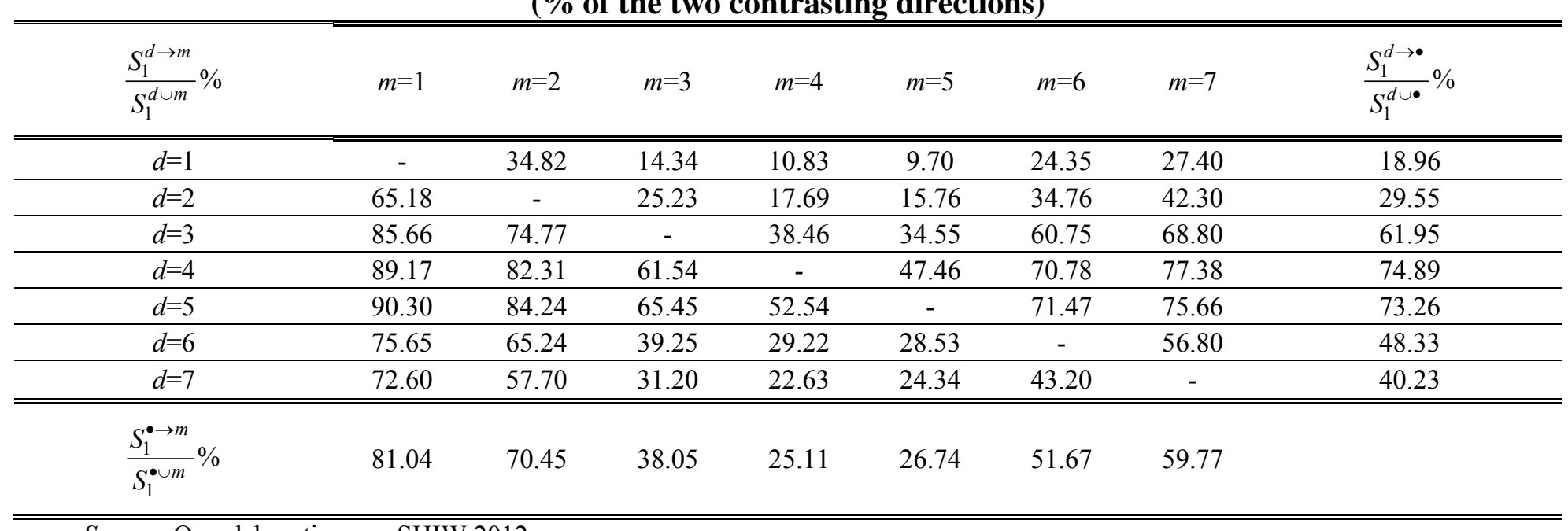

Source: Our elaborations on SHIW 2012. 
Table 4: Axiom 2 - Comparisons of losses between group pairs and between one group and all the others (\% of the two contrasting directions)

\begin{tabular}{|c|c|c|c|c|c|c|c|c|}
\hline$\frac{S_{2}^{d \rightarrow m}}{S_{2}^{d \cup m}} \%$ & $m=1$ & $m=2$ & $m=3$ & $m=4$ & $m=5$ & $m=6$ & $m=7$ & $\frac{S_{2}^{d \rightarrow \bullet}}{S_{2}^{d \cup \bullet}} \%$ \\
\hline$d=2$ & 59.49 & - & 25.65 & 19.26 & 18.59 & 31.78 & 41.89 & 29.42 \\
\hline$d=3$ & 79.21 & 74.35 & - & 40.73 & 41.39 & 55.14 & 67.32 & 60.45 \\
\hline$d=4$ & 82.25 & 80.74 & 59.27 & - & 52.31 & 62.22 & 73.65 & 71.14 \\
\hline$d=7$ & 64.68 & 58.11 & 32.68 & 26.35 & 32.52 & 39.85 & - & 40.80 \\
\hline$\frac{S_{2}^{\bullet \rightarrow m}}{S_{2}^{\bullet \cup m}} \%$ & 74.21 & 70.58 & 39.55 & 28.86 & 35.10 & 46.99 & 59.20 & \\
\hline
\end{tabular}

Source: Our elaborations on SHIW, 2012. 
Table 5: Axiom 3 - Comparisons of losses between group pairs and between one group and all the others (\% of the two contrasting directions)

\begin{tabular}{|c|c|c|c|c|c|c|c|c|}
\hline$\frac{S_{3}^{d \rightarrow m}}{S_{3}^{d \cup m}} \%$ & $m=1$ & $m=2$ & $m=3$ & $m=4$ & $m=5$ & $m=6$ & $m=7$ & $\frac{S_{3}^{d \rightarrow \bullet}}{S_{3}^{d \cup \bullet}} \%$ \\
\hline$d=2$ & 32.69 & - & 74.31 & 83.14 & 85.52 & 63.87 & 57.14 & 70.62 \\
\hline$d=3$ & 14.17 & 25.69 & - & 59.08 & 68.67 & 37.53 & 28.57 & 37.96 \\
\hline$d=4$ & 8.70 & 16.86 & 40.92 & - & 58.21 & 29.40 & 21.91 & 25.62 \\
\hline$d=7$ & 24.32 & 42.86 & 71.43 & 78.09 & 77.83 & 58.33 & - & 61.07 \\
\hline$\frac{S_{3}^{\bullet \rightarrow m}}{S_{3}^{\bullet \cup m}} \%$ & 16.69 & 29.38 & 62.04 & 74.38 & 76.33 & 47.81 & 38.93 & \\
\hline
\end{tabular}

Source: Our elaborations on SHIW, 2012. 
Table 6: Decomposition of the loss of redistributive effect due to axiom violations (\% of corresponding $R E$ )

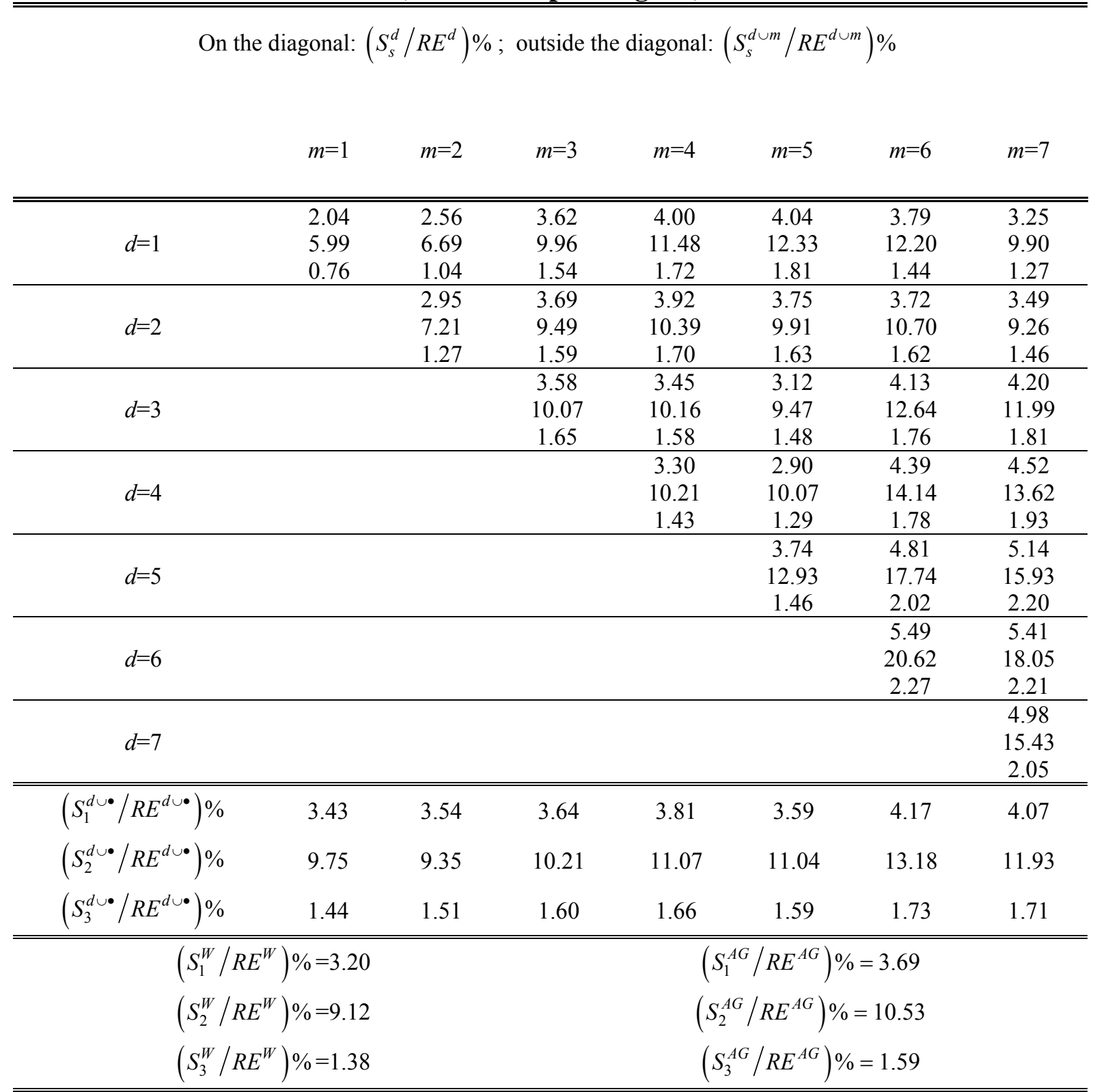

Source: Our elaborations on SHIW, 2012. 


\section{References}

Aronson, J. R., P. Johnson and P. J. Lambert, "Redistributive Effect and Unequal Income Tax Treatment," Economic Journal, 104(423), 262-270, 1994.

Aronson, J. R. and P. J. Lambert, "Decomposing the Gini Coefficient to Reveal Vertical, Horizontal and Reranking Effects of Income Taxation," National Tax Journal, 47(2), 273-294, 1994.

Aronson, J. R., P. J. Lambert and D. R. Trippeer, "Estimates of the Changing Equity Characteristics of the U.S. Income Tax With International Conjectures," Public Finance Review, 27(2), 138-159, 1999.

Auerbach, A. and K. Hassett, “A New Measure of Horizontal Equity," American Economic Review, 92(4), 1116-1125, 2002.

Bank of Italy, Household Income and Wealth in 2010, Supplements to the Statistical Bulletin, XXII (New Series), No. 6, 2012.

Dagum, C., "A New Approach to the Decomposition of Gini Income Inequality Ratio," Empirical Economics, 22(4), 515-531, 1997.

Dardanoni, V. and P. J. Lambert, "Horizontal Inequity Comparisons," Social Choice and Welfare, 18(4), 799-816, 2001.

Duclos, J.-Y., "Gini Indices and the Redistribution of Income," International Tax and Public Finance, 7(2), 141-162, 2000.

Ebert, U., "The Decomposition of Inequality Reconsidered: Weakly Decomposable Measures," Mathematical Social Sciences, 60(2), 94-103, 2010.

Ebert, U. and P. Moyes, “Consistent Income Tax Structures When Households Are Heterogeneous," Journal of Economic Theory, 90(1), 116-150, 2000.

Frosini, B. V., "Approximation and Decomposition of Gini, Pietra-Ricci and Theil Inequality Measures," Empirical Economics, 43(1), 175-197, 2012.

Hyun, J. K. and B.-I. Lim, "Redistributive effect of Korea's income tax: equity decomposition," Applied Economics Letters, 12(3), 195-198, 2005. 
Kakwani, N. C., "On The Measurement of Tax Progressivity and Redistributive Effects of Taxes with Applications to Horizontal and Vertical Equity", in R. L. Basmann and G. F. Rhodes, ed., Advances in Econometrics, Vol. 3, Economics Inequality: Measurement and Policy, 149-168, JAI Press, London, 1984.

Kakwani, N. C. and P. J. Lambert, "On Measuring Inequality in Taxation: A New Approach," European Journal of Political Economy, 14(2), 369-380, 1998.

Kaplow, L., "Horizontal Equity: Measures in Search of a Principle," National Tax Journal, 42(2), 139-154, 1989.

Kaplow, L. and S. Shavell, Fairness versus Welfare, Harvard University Press, Cambridge (MA) and London, 2002.

Lambert, P. J., "Income Taxation and Equity," Baltic Journal of Economics, 4(4), 39$54,2004$.

Ministry of Economics and Finance, Department of Finance, Statistical Reports, 2011. Monti, M. G., "A Note on the Decomposition of the Residual Term of the Gini Index," Argumenta Economica, 20(1), 107-138, 2008.

Monti, M. G., M. Mussini and A. Vernizzi, "The Decomposition of the AtkinsonPlotnick-Kakwani Re-ranking Measure," Statistica Applicata - Italian Journal of Applied Statistics, 22(2), 135-156, 2010.

Pellegrino, S., M. Piacenza and G. Turati, "Developing a Static Micro-simulation Model for the Analysis of Housing Taxation in Italy," The International Journal of Microsimulation, 4(2), 73-85, 2011.

Pellegrino, S. and A. Vernizzi, "On Measuring Violations of the Progressive Principle in Income Tax Systems," Empirical Economics, forthcoming, online first 2012, doi: 10.1007/s00181-012-0613-1.

Urban, I. and P. J. Lambert, "Redistribution, Horizontal Inequity and Re-ranking: How to Measure Them Properly,” Public Finance Review, 36(5), 563-587, 2008. 
van de Ven, J., J. Creedy and P. J. Lambert, "Close Equals and Calculation of the Vertical, Horizontal and Reranking Effects of Taxation," Oxford Bulletin of Economics and Statistics, 63(3), 381-394, 2001.

Wagstaff, A., E. van Doorslaer, H. van der Burg, S. Calonge, T. Christiansen, G. Citoni, U.-G. Gerdtham, M. Gerfin, L. Gross, U. Häkinnen, J. John, P. Johnson, J. Klavus, C. Lachaud, J. Lauridsen, R. E. Leu, B. Nolan, E. Peran, C. Propper, F. Puffer, L. Rochaix, M. Rodríguez, M. Schellhorn, G. Sundberg and O. Winkelhake, "Redistributive Effect, Progressivity and Differential Tax Treatment: Personal Income Taxes in Twelve OECD Countries," Journal of Public Economics, 72(1), 73-98, 1999. 